\title{
Lesões expansivas da órbita: distribuição de casos com estudo histopatológico em 11 anos no Hospital das Clínicas da FMUSP
}

\author{
Orbital space-occupying lesions: an 11-year study of cases with histopathologic \\ analysis seen at Hospital das Clínicas of FMUSP
}

\author{
Hélio Angotti Neto ${ }^{1}$ \\ Leonardo Provetti Cunha ${ }^{2}$ \\ Fábio Gasparin ${ }^{3}$ \\ Ruth Miyuki Santo ${ }^{4}$ \\ Mário Luiz Ribeiro Monteiro ${ }^{5}$
}

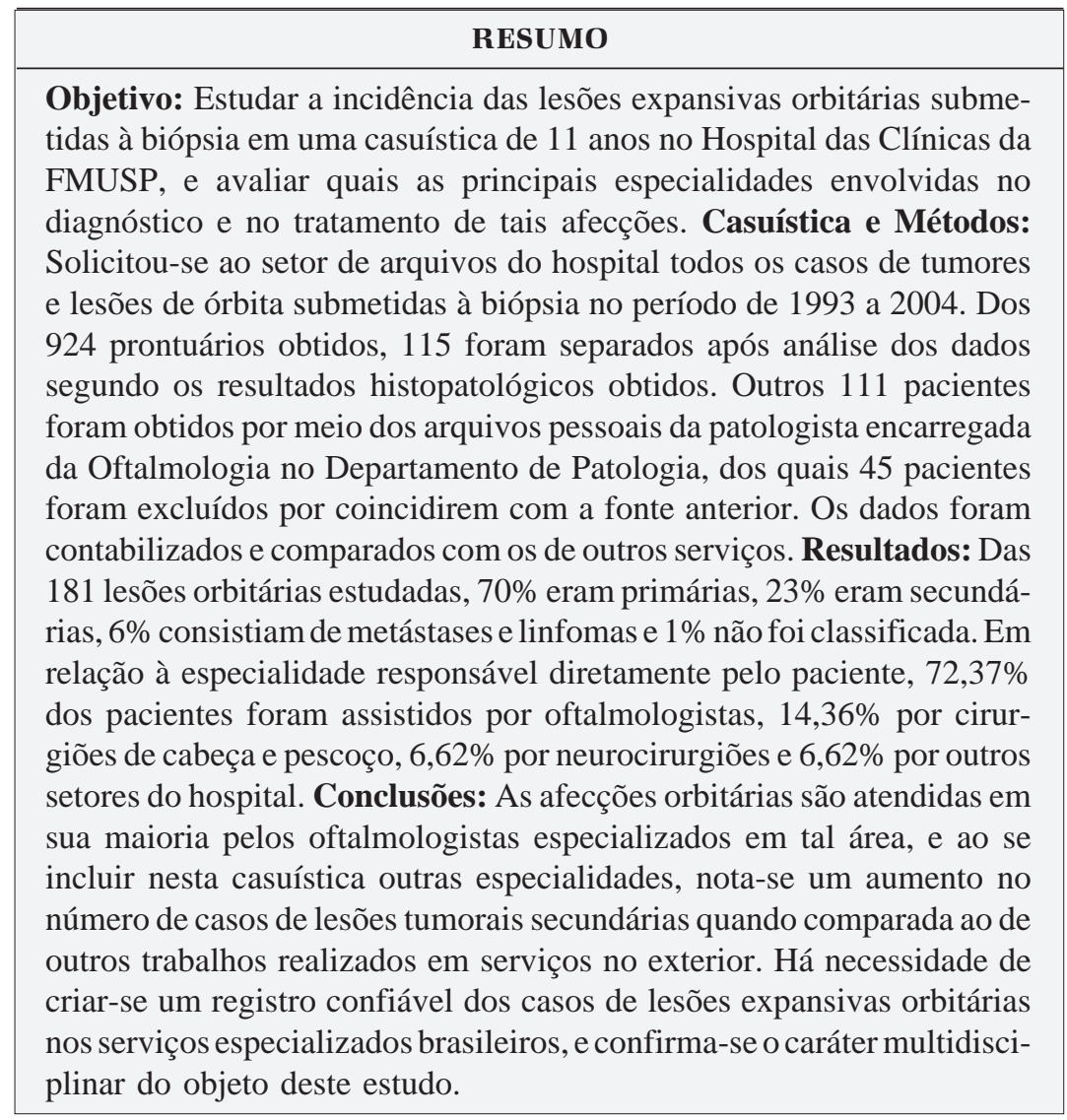

Descritores: Tumores; Órbita/patologia; Neoplasias orbitárias/epidemiologia

\section{INTRODUÇ̃̃̃O}

As afecções orbitárias se incluem entre os mais difíceis diagnósticos dentro da oftalmologia. A dificuldade diagnóstica se deve à heterogeneidade das lesões, incluindo uma grande variedade de afecções inflamatórias, tumorais, congênitas e vasculares ${ }^{(1)}$ e ao pouco conhecimento e estudo dedicados às características e principais apresentações das mesmas pelos oftalmologistas gerais. Em decorrência disto, além das características clínicas e patológicas das doenças orbitárias, é extremamente importante conhecer a distribuição relativa dos diferentes processos patológicos da órbita. 
Apesar da necessidade, poucos são os estudos epidemiológicos a respeito, a maioria deles realizada em instituições no exterior. Shields publicou em 2004 uma casuística de 1.264 tumores orbitários reunidos durante 30 anos no Will's Eye Hospital ${ }^{(2)}$. Lee, em Singapura, reuniu uma casuística de 125 tumores oculares, dos quais 19 envolviam órbitas e glândulas lacrimais, reunidos num período de $17 \operatorname{anos}^{(3)}$. Na Suécia, Seregard e Sahlin revisaram 24 anos de atendimento num serviço terciário, reunindo 300 casos de lesões orbitárias operadas ou biopsiadas ${ }^{(4)}$. Johansen, da Dinamarca, também reuniu uma casuística de 24 anos, obtendo um número total de 965 lesões orbitárias em 841 pacientes $^{(5)}$.

Outra dificuldade no estudo das doenças da órbita se deve ao caráter multidisciplinar envolvido no diagnóstico e no tratamento das mesmas. Muitas das lesões orbitárias afetam estruturas diversas, seja por meio de metástases ou por contigüidade, e vice-versa, exigindo uma ação conjunta do oftalmologista com especialistas de outras áreas como neurocirurgiões, otorrinolaringologistas, cirurgiões plásticos, cirurgiões de cabeça e pescoço e dermatologistas, que muitas vezes são os primeiros a receber e tratar o paciente com uma queixa de alteração na órbita. Nenhum dos trabalhos citados anteriormente se preocupou em definir o local de atendimento inicial e internação do paciente, ou qual especialidade o assistiu num primeiro momento.

O objetivo deste trabalho é avaliar a incidência das lesões expansivas orbitárias operadas ou biopsiadas num grande serviço hospitalar terciário, assim como identificar quais foram os setores do hospital nos quais os pacientes foram internados, no sentido de melhor compreender o caráter multidisciplinar da investigação diagnóstica e da terapêutica das afecções orbitárias.

\section{MÉTODOS}

Foi solicitada ao setor de arquivos do Hospital das Clínicas da FMUSP a listagem de pacientes, com diagnóstico de tumor de órbita ou lesão orbitária a ser biopsiada, internados naquele hospital entre 1993 e 2004. Por meio da listagem de pacientes obtida com o Sistema de Estatística Médica, acessou-se o sistema computadorizado do hospital e foram obtidos os dados referentes aos resultados dos estudos histopatológicos dos mesmos.

Dos 924 prontuários levantados, foram incluídos no presente estudo todos aqueles pacientes que apresentavam biópsia ou exérese de massas orbitárias segundo os dados disponíveis, totalizando 115 pacientes. Outros dados coletados foram a idade do paciente, sua data de internação, sexo e setor do hospital no qual foi admitido.

Uma segunda fonte de dados foi a pesquisa realizada nos arquivos pessoais da médica patologista encarregada pela avaliação dos estudos histopatológicos relacionados à Oftalmologia e Órbita, acrescentando um número de 111 pacientes, dos quais 45 se repetiam quando as duas fontes foram pareadas. Foram então obtidos 181 casos de massas orbitárias com estudo histopatológico realizados entre 1993 e 2004. Os resultados foram contabilizados e comparados com outras instituições.

\section{RESULTADOS}

Das 181 lesões encontradas, 2 eram tumores metastáticos, 9 linfoproliferativos, 42 eram lesões secundárias e 2, tumores que não foram classificados. Os 126 restantes foram lesões primárias da órbita, incluindo lesões inflamatórias e hemorrágicas (Figura 1). Foram consideradas lesões primárias todas aquelas que se manifestaram primariamente na órbita, enquanto que as secundárias foram aquelas que se originaram de estruturas próximas à órbita, invadindo-a por contigüidade (Ex: tumores faciais, sinusais, conjuntivais, palpebrais etc.).

Do total, 91 estudos histopatológicos pertenciam a pacientes do sexo masculino, enquanto que 90 pertenciam a pacientes do sexo feminino. A média de idade em anos completos foi de 43,2 anos com desvio padrão de 18,8 anos.

Dos tumores classificados como metastáticos, um era originário da tireóide e outro advinha de um carcinoma de células renais. Todos os pacientes com tumores linfoproliferativos foram diagnosticados como linfoma.

A maioria das lesões secundárias correspondeu aos tumores de acometimento palpebral e de pele da face. Somente 1 era retinoblastoma de crescimento intra-ocular com acometimento de tecido extra-ocular. Foram diagnosticados 23 carcinomas epidermóides, 16 carcinomas basocelulares e 2 carcinomas de glândulas sebáceas. Pela falta de dados disponíveis no prontuário e nos estudos histopatológicos não foi possível separar exatamente quais acometiam primariamente a pele e quais acometiam primariamente a conjuntiva.

Das 126 lesões orbitárias primárias obtivemos 31 lesões inflamatórias ou infecciosas (21 lesões inflamatórias inespecíficas, 3 processos inflamatórios crônicos granulomatosos, 6 granulomas de corpo estranho e 1 paciente com mucormicose), 22 tumores císticos (16 cistos dermóides, 5 ectasias duc-

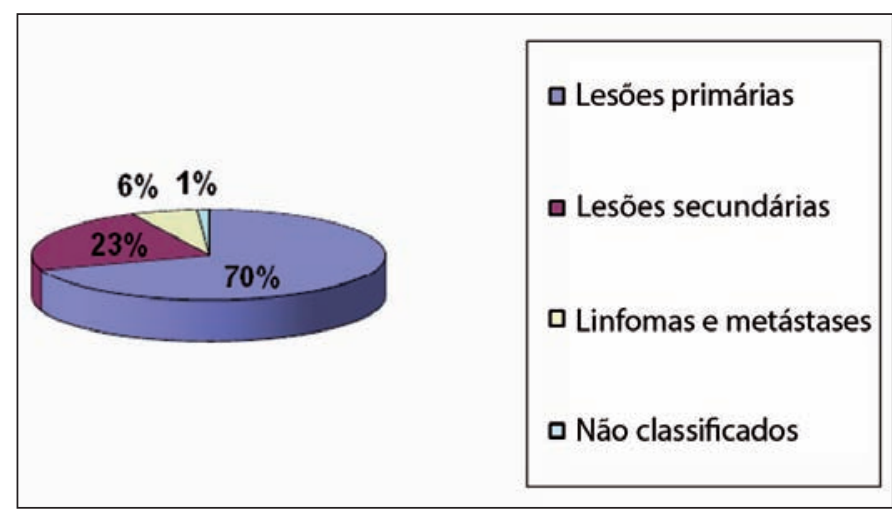

Figura 1 - Proporção quanto à classificação dos 181 tumores orbitários com confirmação histopatológica estudados no HCFMUSP no período de 11 anos 
tais da glândula lacrimal e 1 cisto de Moll), 19 tumores acometendo glândulas lacrimais (7 adenomas pleomórficos, 6 adenocarcinomas, 3 carcinomas adenóides císticos e 3 carcinomas mucoepidermóides), 14 tumores vasculares ( 7 hemangiomas cavernosos, 4 hemangiomas capilares, 2 linfangiomas e 1 hemangiopericitoma), 11 tumores melanocíticos primários (10 melanomas e 1 nevo azul), 7 de nervo óptico e meninge (4 astrocitomas pilocíticos, 2 meningiomas e 1 tumor neuroectodérmico primitivo), 6 de nervos periféricos (3 Schwanomas e 3 neurofibromas), 5 miogênicos (4 rabdomiossarcomas e 1 leiomiossarcoma), 3 de tecido ósseo (1 displasia fibrosa, 1 tumor marrom e 1 aneurisma cirsóide) e 2 de tecido fibroso e de tecido conectivo (fibro-histiocitoma e lipoma), 1 cisto hemorrágico, 1 hematoma, 1 disgerminoma, 2 teratomas e 1 paraganglioma. Os dados encontram-se dispostos na tabela 1 com porcentagens para melhor visualização.

A faixa pediátrica, aqui considerando todos os pacientes que foram internados com idade menor que ou igual a 18 anos, representou cerca de $17,67 \%$ do total (32 casos). As lesões císticas foram as mais numerosas, totalizando $37,5 \%$ das lesões pediátricos ( 9 cistos dermóides e 3 ectasias ductais da glândula lacrimal). Outras encontradas foram: 3 rabdomiossarcomas (9,37\%), 3 astrocitomas pilocíticos (9,37\%), 2 lesões inflamatórias não específicas $(6,25 \%), 1$ retinoblastoma com extensão extra-ocular, 1 linfangioma, 1 melanoma maligno, 1 meningioma, 1 carcinoma basocelular, 1 neurofibroma, 1 Schwanoma, 1 disgerminoma, 1 fibro-histiocitoma maligno e 1 teratoma ( $3,12 \%$ cada).

A maioria dos pacientes esteve internada nas dependências da clínica oftalmológica, totalizando aproximadamente $72,37 \%$ do total (131 pacientes). O restante esteve internado nas seguintes enfermarias: $14,36 \%$ na enfermaria cirúrgica de cabeça e pescoço (26 pacientes), 6,62\% nas enfermarias neurocirúrgicas (12 pacientes), e o restante dividido nas enferma-

\begin{tabular}{lcc|}
$\begin{array}{l}\text { Tabela 1. Distribuição relativa dos } \\
\text { firmação histopatológica estudados no HCFMUSP } \\
\text { 11 anos (1993-2004) }\end{array}$ & Número & $\%$ \\
Tumores orbitários & 42 & 23,2 \\
Tumores secundários & & \\
Lesões inflamatórias & 31 & 17,1 \\
inespecíficas e granulomatosas & 22 & 12,2 \\
Lesões císticas & 19 & 10,5 \\
Tumores de glândula lacrimal & 14 & 7,7 \\
Tumores vasculares & 11 & 6,1 \\
Tumores melanocíticos primários & 9 & 5,0 \\
Linfomas & 7 & 3,9 \\
Tumores de nervo óptico e meninge & 6 & 3,3 \\
Tumores de nervos periféricos & 5 & 2,8 \\
Tumores miogênicos & & \\
Tumores ósseos, cartilaginosos & 5 & 2,8 \\
$\quad$ e do tecido conectivo & 2 & 1,1 \\
Tumores metastáticos & 2 & 1,1 \\
Não classificados & 6 & 3,3 \\
Outros & &
\end{tabular}

rias de Clínica médica (3 pacientes), Dermatologia (2), Otorrinolaringologia (2), Pediatria (2), Cirurgia Plástica (1), Hematologia (1) e Pronto-Socorro (1) (Figura 2).

A segunda especialidade mais freqüentemente envolvida com os tumores orbitários, após a Oftalmologia, foi a de Cirurgia de Cabeça e Pescoço, na qual, as afecções orbitárias tratadas consistiram de 10 carcinomas epidermóides, 10 carcinomas basocelulares, 3 carcinomas adenóides císticos, 1 metástase de carcinoma anaplásico de tireóide, 1 granuloma de corpo estranho e 1 carcinoma de glândulas sebáceas (Figura 2).

\section{DISCUSSÃO}

Os tumores orbitários são relativamente raros na população geral, totalizando aproximadamente $8,8 \%$ dos tumores oftálmicos ${ }^{(3)}$. Além disso, a maioria das casuísticas obtidas sofre diversos vieses como a origem dos dados obtidos ou a instituição abordada. A casuística publicada por Seregard, da Suécia, baseou-se em 300 pacientes atendidos num serviço especializado em oftalmologia no decorrer de $24 \operatorname{anos}^{(4)}$, assim como a de Shields, que utilizou os dados provenientes de um hospital especializado em oftalmologia na Filadélfia reunindo um total de 645 casos no período de 20 anos $^{(2)}$. Já outros trabalhos se basearam em bancos de dados mais abrangentes. Em um trabalho dinamarquês ${ }^{(5)}$ foram acessados todos os departamentos de patologia daquele país, enquanto que no trabalho realizado em Singapura ${ }^{(3)}$ foi usado como fonte o Registro de Câncer de Singapura.

Este trabalho, embora limitado a uma instituição, tem a peculiaridade de identificar outras especialidades envolvidas com o diagnóstico e tratamento das afecções orbitárias e adicionar suas casuísticas ao total. Provavelmente foi esta característica que resultou em um alto índice de lesões neoplásicas secundárias, totalizando $23,2 \%$ do total, enquanto que outros estudos mostraram casuísticas que variavam de 11 a $19,8 \%{ }^{(2,4-5)}$. A especialidade que mais internou pacientes com tumores orbitários após a oftalmologia, neste estudo, foi cirurgia de cabeça e pescoço, contribuindo com um total de $14,36 \%$ dos casos estudados.

Embora o número de lesões metastáticas (excluídos os linfomas) seja menor que o de outros trabalhos, totalizando $1,1 \%$ do total, é certo que não representa o real valor numérico de lesões metastáticas em órbita, já que muitos tumores são diag-

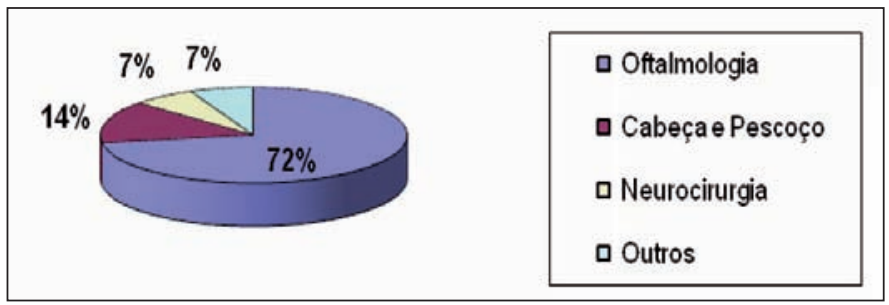

Figura 2 - Proporção dos 181 tumores orbitários quanto à especialidade primariamente envolvida no tratamento 
nosticados por meio de biópsias em outros sítios ou criteriosa investigação clínica, fontes que não foram acrescentadas nesta casuística.

$\mathrm{Na}$ faixa pediátrica, assim como no trabalho publicado por Shields, a lesão expansiva mais encontrada foi a de caráter cístico. Em nosso estudo, tais lesões totalizaram 37,5\% dos casos na infância. No trabalho de Shields, que reuniu 250 casos na faixa pediátrica, estudados de junho de 1962 a dezembro de 1983 , as lesões císticas alcançaram $52 \%$ do total ${ }^{(6)}$.

As lesões primárias de órbita representaram a maioria dos diagnósticos realizados pelo estudo histopatológico, totalizando $63,29 \%$ do total das 181 lesões estudadas.

Deve ser lembrado que, assim como nas casuísticas previamente citadas, este trabalho sofreu diversos vieses. Os dados obtidos vieram de uma instituição pública de caráter terciário que sofreu uma série de mudanças com o passar dos anos em sua armazenagem de dados e que está submetida a diversos fatores limitantes como o mau preenchimento de prontuários ou a perda dos mesmos. Um exemplo ilustrativo é o elevado número de resultados histopatológicos (66 casos) obtidos pelos registros da patologista especializada em órbita e oftalmologia e que não foram obtidos na pesquisa ao banco de dados informatizado. Outros fatores limitantes deste trabalho se devem à falta de inclusão na casuística de lesões expansivas não submetidas a procedimentos cirúrgicos, que foram apenas acompanhados clinicamente no serviço ambulatorial de órbita ou de outras especialidades, o que subestima grandemente a casuística total das lesões orbitárias diagnosticadas e tratadas no Hospital das Clínicas; e à ausência de um arquivo no setor de órbita dedicado ao registro e histórico dos pacientes atendidos até o ano de conclusão desta pesquisa.

Uma informação nova que recebeu pouca atenção nos outros trabalhos foi a inclusão dos dados referentes ao serviço de origem dos pacientes estudados, confirmando o caráter multidisciplinar do estudo das afecções orbitárias. A oftalmologia mostrou-se como a mais freqüentemente envolvida num primeiro atendimento e na internação do paciente e, portanto, do nosso ponto de vista, a com maior responsabilidade ao lidar com tais afecções. Como durante a internação ocorre na instituição de forma dinâmica a troca de informações e a colaboração entre as diversas especialidades envolvidas, não foi possível avaliar a real extensão da participação de cada especialidade durante o tratamento. Também não se pode dizer que tenha havido em todos os casos a interação adequada entre o setor de oftalmologia e as demais clinicas envolvidas, embora a cooperação multidisciplinar tanto no diagnóstico como no tratamento tenha existido na maioria dos casos.

O presente estudo serve para enfatizar a necessidade de se criar um registro confiável das afecções tumorais oftálmicas, não somente em nosso serviço, mas nos principais centros de tratamento oftalmológico em todo o Brasil, a fim de termos um real espelho da situação do país no que se refere a uma condição potencialmente letal e muitas vezes mutiladora. O estudo das doenças orbitárias é uma responsabilidade que o oftalmologista geral não deve recusar. Tal estudo tem que ser estimulado para o profissional que é percebido pela população como o responsável principal no trato das afecções orbitárias e não deve se omitir desta atribuição.

\section{ABSTRACT}

Purpose: To report the incidence of orbital space-occupying lesions in an 11-year interval study of patients examined at the Hospital das Clínicas of FMUSP, and to evaluate which are the main specialties involved in the diagnosis and treatment of such conditions. Methods: All cases of orbital spaceoccupying lesions in the period from 1993 to 2004 were revised. Of 924 obtained medical records, 155 were selected after histopathological analysis. Another 111 cases were gathered from the personal archives of a pathologist responsible for Ophthalmic Histopathology, from which 45 cases were excluded because they had already been included in the first source analysis. The data were analyzed and compared with the results from other institutions. Results: Of the studied 181 orbital space-occupying lesions, $70 \%$ were primary, $23 \%$ secondary, 6\% metastatic and lymphomas, and 1\% was not classified. The most common specialties involved ophthalmologists in $72.37 \%$ of all 181 cases, head and neck surgeons in $14.36 \%$, neurosurgeons in $6.62 \%$, and others in $6.62 \%$. Conclusions: The orbital mass lesions were treated mostly by an expert ophthalmologist. When other specialties were included, there was a modest increase in secondary tumor incidence when the final result was compared with previous studies on this subject. Our findings indicate that the creation of a trustworthy register of all orbital space-occupying lesions in Brazilian specialized services is necessary. The multidisciplinary character of the orbital mass lesions is corroborated by this review.

Keywords: Tumors; Orbit/pathology; Orbital neoplasms/ epidemiology

\section{REFERÊNCIAS}

1. Dantas AM, Monteiro MLR. Doenças da órbita. Rio de Janeiro: Cultura Médica; 2002

2. Shields JA, Shields CL, Scartozzi R. Survey of 1264 patients with orbital tumors and simulating lesions: The 2002 Montgomery Lecture, part 1. Ophthalmology. 2004;111(5):997-1008.

3. Lee SB, Au Eong KG, Saw SM, Chan TK, Lee HP. Eye cancer incidence in Singapore. Br J Ophthalmol. 2000;84(7):767-70.

4. Seregard S, Sahlin S. Panorama of orbital space-occupying lesions. The 24 year experience of a referral centre. Acta Ophthalmol Scand. 1999;77(1):91-8.

5. Johansen S, Heegaard S, Bogeskov L, Prause JU. Orbital space-occupying lesions in Denmark 1974-1997. Acta Ophthalmol Scand. 2000;78(5):547-52.

6. Shields JA, Bakewell B, Augsburger JJ, Donoso LA, Bernardino V. Spaceoccupying orbital masses in children. A review of 250 consecutive biopsies. Ophthalmology. 1986;93(3):379-84. 